\title{
COMPLICAÇÕES PÓS-OPERATÓRIAS DE COLECISTECTOMIAS - ANÁLISE COMPARATIVA EM RELAÇÃO AO SEXO
}

\author{
POSTOPERATIVE COMPLICATIONS OF CHOLECYSTECTOMIES - \\ COMPARATIVE ANALYSIS RELATED TO GENDER
}

\author{
Roberto Mariano Gómez Hangui ${ }^{1}$ \\ Ronaldo Elías Carnut Rêgo, TCBC-SP ${ }^{2}$ \\ Viviane Coimbra Augusto Demarchi ${ }^{3}$ \\ Flávio Daniel Saavedra Tomasich, TCBC-PR ${ }^{4}$ \\ Adhemar M. Pacheco Jr., TCBC-SP ${ }^{5}$
}

\begin{abstract}
RESUMO: Objetivo: Analisar a incidência das complicações pós-operatórias das colecistectomias por via aberta e laparoscópica, em relação ao sexo. Método: Estudo retrospectivo de 1123 pacientes submetidos a colecistectomia eletiva, por via aberta ou laparoscópica, no Departamento de Cirurgia da Santa Casa de Misericórdia de São Paulo, no período de Janeiro/1997 a Janeiro/2001. Foi realizada uma avaliação comparativa das complicações pós-operatórias, relacionando os resultados com a idade, ASA, via de acesso, tempo de cirurgia, acidentes intra-operatórios, necessidade de drenagem, período de permanência hospitalar, resultado anátomo-patológico e mortalidade. Resultados: Houve predominância do sexo feminino na população estudada, com 82,5\% dos casos. A média de idade foi de 48,8 anos (14 a 97 ). Das operações realizadas, 693 $(61,7 \%)$ foram por via laparoscópica, sendo a maior proporção de abordagens abertas no sexo masculino $(\mathrm{p}=0,0014)$. A taxa global de conversão foi de $2,31 \%$, sem diferença entre os sexos, assim como a realização de procedimentos associados e a ocorrência de acidentes intra-operatórios. A duração da intervenção, a drenagem, a permanência hospitalar pós-operatória, complicações e mortalidade foram significativamente mais freqüentes entre os homens. Neste grupo, observou-se duração maior do ato operatório e permanência hospitalar pós-operatória, com significância estatística, principalmente quando se realizou o tratamento tradicional. Conclusões: O sexo masculino mostrou-se como provável fator de risco para complicações nas colecistectomias, resultando em operações prolongadas, maior período de permanência hospitalar, necessidade de drenagem mais freqüente e mortalidade elevada em relação ao sexo feminino.
\end{abstract}

Descritores: Colecistectomia; Complicações pós-operatórias; Sexo.

\section{INTRODUÇÃO}

A idade é um importante fator preditivo de complicações após colecistectomia pela incidência aumentada de doença do trato biliar complicada e pela maior morbi-mortalidade da cirurgia no idoso, em de- corrência das doenças associadas ${ }^{1-3}$. Entretanto, poucos trabalhos enfocam o papel do sexo ou uma possível interação deste com a idade nas complicações pósoperatórias de pacientes com doença litiásica.

Numa análise de regressão logística multivariada com 30145 pacientes, Russell et $a l^{2}$ de-

1. $\quad$ Ex-residente do Departamento de Cirurgia da F.C.M.S.C.S.P.

2. Mestre em Cirurgia Geral do Departamento de Cirurgia da F.C.M.S.C.S.P.

3. Ex-Residente em Cirurgia Oncológica do Hospital Erasto Gaertner; Aluna do Curso de Pós-Graduação em Cirurgia - Nível Mestrado da F.C.M.S.C.S.P.

4. Médico Titular do Serviço de Cirurgia Abdominal do Hospital Erasto Gaertner; Aluno do Curso de Pós-Graduação em Cirurgia Nível Doutorado da F.C.M.S.C.S.P.

5. Professor Adjunto e Chefe do Grupo de Vias Biliares do Departamento de Cirurgia da F.C.M.S.C.S.P.

Recebido em 06/06/2003

Aceito para publicação em 21/11/2003

Trabalho realizado no Departamento de Cirurgia da Faculdade de Ciências Médicas da Santa Casa de São Paulo (F.C.M.S.C.S.P.). 
monstraram que o grupo masculino submetido a colecistectomia apresenta doença biliar mais grave. Observou-se um risco aumentado de conversão para cirurgia aberta e maior mortalidade pós-operatória nesse segmento. Habitualmente homens submetemse com maior frequiência ao tratamento clínico nos surtos de agudização, postergando a intervenção cirúrgica e resultando na elevação da morbi-mortalidade. Os autores defendem uma conduta mais intervencionista da doença litiásica sintomática em homens, principalmente em idosos.

O objetivo deste estudo é analisar a incidência das complicações pós-operatórias das colecistectomias realizadas no Departamento de Cirurgia da Santa Casa de São Paulo, em relação ao sexo, relacionando com características clínicas e cirúrgicas.

\section{MÉTODO}

Analisamos retrospectivamente 1123 pacientes submetidos a colecistectomia por doença litiásica, admitidos no Departamento de Cirurgia Geral da Santa Casa de Misericórdia de São Paulo, no período de janeiro de 1997 a janeiro de 2001. Foram incluídos tanto os procedimentos tradicionais quanto por via laparoscópica, sendo classificados como "conversão" todos aqueles inicialmente operados por vídeo-cirurgia e necessitaram mudança para via aberta no intraoperatório. Foram excluídas as colecistectomias realizadas por trauma, por doença neoplásica pancreática ou biliar, as realizadas em associação com operações abdominais simultâneas (exceto da via biliar), ou por outras indicações.
Para a coleta dos dados foi utilizado um protocolo individual, e foram avaliadas as seguintes variáveis: sexo, idade, passado sintomático, risco anestésico (A.S.A. - American Society of Anesthesiology), via de acesso cirúrgico, taxa de conversão, tipo de operação realizada, tempo cirúrgico, intercorrências ou seqüelas das operações, necessidade de drenagem, permanência hospitalar após as intervenções, complicações, mortalidade pós-operatória e exame anátomopatológico da peça ressecada. Considerou-se como acidentes intra-operatórios a ocorrência de sangramento, lesões de via biliar, hepática e/ou duodenal.

Aos resultados aplicou-se análise estatística descritiva e específica para cada variável, e a avaliação das possíveis associações entre as variáveis foi feita através dos testes de diferença entre médias, de proporções e qui-quadrado. $O$ nível de significância adotado foi de $\mathrm{p}<0,05$.

\section{RESULTADOS}

Entre primeiro de janeiro de 1997 e 30 de janeiro de 2001, realizaram-se 1123 colecistectomias por doença litiásica, sendo $930(82,8 \%)$ doentes do sexo feminino e 193 (17,2\%) do masculino. A distribuição dos casos é sumarizada na Tabela 1 e a Tabela 2 mostra as variáveis relacionadas ao sexo.

As conversões deveram-se a: dificuldade técnica $(50 \%)$, coledocolitíase $(26,9 \%)$, lesão de colédoco $(11,5 \%)$, sangramento $(7,7 \%)$ e fístula colecistoduodenal $(3,8 \%)$, totalizando 26 casos. Nos homens, observaram-se apenas dois casos, sendo um por difi-

Tabela 1 - Distribuição dos doentes $(n=1123)$.

\begin{tabular}{|c|c|c|c|c|}
\hline & Total $(n=1123)$ & Feminino $(n=930)$ & Masculino $(n=193)$ & Significância \\
\hline Idade (em anos) & $\begin{array}{c}14-97 \\
(\text { média }=48,8)\end{array}$ & $\begin{array}{l}14-97 \\
(\text { média }=47,6)\end{array}$ & $\begin{array}{l}20-87 \\
(\text { média }=54,8)\end{array}$ & $\mathrm{p}=0,0000$ \\
\hline \multicolumn{5}{|c|}{ Tempo de sintomatologia } \\
\hline 3-6 meses & $(11,7 \%)$ & $(11,4 \%)$ & $(13 \%)$ & NS \\
\hline$>6$ meses & $(77,7 \%)$ & $(78,6 \%)$ & $(73,6 \%)$ & NS \\
\hline \multicolumn{5}{|c|}{ Risco anestésico (ASA) } \\
\hline I & $(48 \%)$ & $(49 \%)$ & $(43,5 \%)$ & NS \\
\hline II & $(49,7 \%)$ & $(49,2 \%)$ & $(51,8 \%)$ & NS \\
\hline III & $(2,4 \%)$ & $(1,8 \%)$ & $(4,7 \%)$ & $\mathrm{p}=0,0146$ \\
\hline
\end{tabular}


Tabela 2 - Variáveis relacionadas ao sexo.

\begin{tabular}{|c|c|c|c|c|c|c|c|}
\hline \multirow{2}{*}{ Via inicial de acesso cirúrgico } & \multicolumn{2}{|c|}{ Total $(n=1123)$} & \multicolumn{2}{|c|}{ Feminino $(n=930)$} & \multicolumn{2}{|c|}{ Masculino $(n=193)$} & \multirow[t]{2}{*}{ Significância } \\
\hline & & & & & & & \\
\hline Aberta & 430 & $(38,3 \%)$ & 336 & $(36,1 \%)$ & 94 & $(48,7 \%)$ & $p=0,0014$ \\
\hline Laparoscópica & 693 & $(61,7 \%)$ & 594 & $(63,9 \%)$ & 99 & $(51,3 \%)$ & \\
\hline Cirurgias associadas & 31 & $(2,7 \%)$ & 23 & $(2,5 \%)$ & 8 & $(4,2 \%)$ & NS \\
\hline $\begin{array}{l}\text { Conversão para } \\
\text { cirurgia aberta }\end{array}$ & 26 & $(2,3 \%)$ & 24 & $(2,5 \%)$ & 2 & $(1,0 \%)$ & NS \\
\hline Tempo de cirurgia (em minutos) & $136=$ & 67 & 133 & +65 & 155 & \pm 76 & $\mathrm{p}=0,0004$ \\
\hline Aberta & $168=$ & 65 & 157 & +66 & 186 & +84 & $\mathrm{p}=0,0000$ \\
\hline Laparoscópica & $114=$ & 61 & 119 & \pm 61 & 119 & \pm 50 & NS \\
\hline \multicolumn{8}{|l|}{ Permanência hospitalar } \\
\hline pós-operatória (em dias) & \multicolumn{2}{|c|}{$2,0 \pm 2,4$} & \multicolumn{2}{|c|}{$1,8 \pm 1,6$} & \multicolumn{2}{|c|}{$2,7 \pm 4,5$} & $\mathrm{p}=0,0000$ \\
\hline Aberta & \multicolumn{2}{|c|}{$2,6 \pm 3,2$} & \multicolumn{2}{|c|}{$2,4 \pm 2,7$} & \multicolumn{2}{|c|}{$3,7 \pm 5,5$} & $\mathrm{p}=0,0010$ \\
\hline Laparoscópica & \multicolumn{2}{|c|}{$1,5 \pm 1,5$} & \multicolumn{2}{|c|}{$1,5 \pm 1,0$} & \multicolumn{2}{|c|}{$1,8 \pm 3,0$} & $\mathrm{NS}$ \\
\hline Acidentes intraoperatórios & 101 & $(9,0 \%)$ & 79 & $(8,5 \%)$ & 22 & $(11,4 \%)$ & NS \\
\hline Aberta & 39 & $(9,1 \%)$ & 27 & $(8,0 \%)$ & 12 & $(12,8 \%)$ & \\
\hline Laparoscópica & 62 & $(8,9 \%)$ & 52 & $(8,8 \%)$ & 10 & $(10,1 \%)$ & \\
\hline Drenagem & 341 & $(30,4 \%)$ & 266 & $(28,6 \%)$ & 75 & $(38,8 \%)$ & $\mathrm{p}=0,0047$ \\
\hline Aberta & 117 & $(27,2 \%)$ & 76 & $(22,6 \%)$ & 41 & $(43,6 \%)$ & \\
\hline Laparoscópica & 224 & $(32,3 \%)$ & 187 & $(31,5 \%)$ & 37 & $(37,4 \%)$ & \\
\hline Complicações & 39 & $(3,5 \%)$ & 26 & $(2,8 \%)$ & 13 & $(6,7 \%)$ & $\mathrm{p}=0,0072$ \\
\hline Aberta & 21 & $(4,9 \%)$ & 10 & $(3,0 \%)$ & 11 & $(11,7 \%)$ & \\
\hline Laparoscópica & 17 & $(2,5 \%)$ & 16 & $(2,7 \%)$ & 1 & $(1,0 \%)$ & \\
\hline Mortalidade pós-operatória & 5 & $(0,4 \%)$ & 1 & $(0,1 \%)$ & 4 & $(2,1 \%)$ & $\mathrm{p}=0,000$ \\
\hline Aberta & 4 & $(0,9 \%)$ & 0 & & 4 & $(4,3 \%)$ & \\
\hline Laparoscópica & 1 & $(0,1 \%)$ & 1 & $(0,2 \%)$ & 0 & & \\
\hline
\end{tabular}

culdade técnica e outro por fístula colecisto-duodenal. No grupo feminino, entre as 24 conversões anotaramse as seguintes causas: dificuldade técnica em 12 pacientes (50\%), coledocolitíase em sete $(29,2 \%)$, lesão de colédoco em três $(12,5 \%)$ e sangramento em dois casos $(8,3 \%)$.

Realizada exploração de vias biliares em 31 pacientes, resultando em três papilotomias transduodenais e oito derivações bilio-digestivas, das quais sete coledoco-duodenoanastomoses e uma hepato-jejunoanastomose.
Em relação aos acidentes intraoperatórios observados, temos em ordem de freqüência decrescente: 90 casos $(8 \%)$ de sangramento, sete $(0,6 \%)$ lesões de via biliar, três $(0,3 \%)$ lesões hepáticas e uma $(0,1 \%)$ lesão duodenal. Quando estratificados por sexo, anotaram-se respectivamente em homens e mulheres: $21(10,8 \%)$ e $69(7,4 \%)$ casos de sangramento, uma $(0,5 \%)$ e seis $(0,6 \%)$ lesões de via biliar, sendo os casos de lesão hepática - três $(1,5 \%)$ e lesão duodenal - uma $(0,5 \%)$, observados exclusivamente em mulheres. 
Os resultados dos estudos anátomo-patológicos mostraram $92,2 \%$ de colecistite crônica e $6,9 \%$ de aguda. Os $0,9 \%$ restantes representam outros diagnósticos.

Trinta e nove pacientes (3,5\%) evoluíram com complicações, sendo $13(6,7 \%)$ do sexo masculino e $26(2,8 \%)$ do feminino, com diferença estatisticamente significante através do teste de proporções $(\mathrm{p}=0,0072)$. As complicações anotadas são especificadas na Tabela 3.

Tabela 3 - Complicações pós-operatórias.

\begin{tabular}{lll}
\hline Complicações pós-operatórias & N $(\%)$ \\
\hline Fístula biliar & 8 & $(20,5)$ \\
Pancreatite aguda & 6 & $(15,4 \%)$ \\
Broncopneumonia & 4 & $(10,3 \%)$ \\
Perda do dreno & 3 & $(7,7 \%)$ \\
Evisceração & 3 & $(7,7 \%)$ \\
Íleo prolongado & 3 & $(7,7 \%)$ \\
Coleção intra-abdominal & 3 & $(7,7 \%)$ \\
Acidente vascular cerebral & 2 & $(5,1 \%)$ \\
Outros & 7 & $(17,9 \%)$ \\
\hline
\end{tabular}

A mortalidade operatória foi de $0,4 \%$. Dentre os cinco casos de óbito, quatro $(2,1 \%)$ foram do sexo masculino e $1(0,1 \%)$ do feminino, sendo as causas de morte: pancreatite grave $(1,6 \%)$, abdome agudo vascular $(0,5 \%)$, peritonite pós-reparo de lesão duodenal $(0,1 \%)$.

\section{DISCUSSÃO}

A doença litiásica biliar nas mulheres é predominante na faixa etária abaixo dos 60 anos, mas a associação do sexo em relação às características da doença e à cirurgia, assim como a sua evolução, são pouco discutidas na literatura. Entretanto, Russell et $a l^{2}$ demonstraram uma relação do sexo e da idade superior a 65 anos com doença complicada e maior risco de mortalidade pós-operatória.

A frequiência do elemento feminino nos pacientes submetidos a colecistectomia varia de 61,9 a $90 \%{ }^{4-7}$. Essa característica epidemiológica confirmou-se neste estudo, no qual $82,8 \%$ da amostra era constituída de mulheres, com uma relação feminino: masculino de 4,8:1.

Apesar do predomínio da doença e do tratamento cirúrgico no grupo feminino, a ocorrência de colecistite aguda e a mortalidade são significativamente maiores nos homens em diversos estudos, parecendo demonstrar uma doença com evolução natural diferente nos dois sexos.

Sanabria $e t$ al $^{8}$ comentaram em seu estudo que o grau de inflamação no quadrante superior direito em homens sintomáticos é significativamente maior que o observado nas mulheres, especulando que esse fato poderia ser explicado pela demora da população masculina em procurar atendimento médico. O tempo de sintomatologia ao diagnóstico em nosso estudo não mostrou diferença significativa entre os grupos, o que contradiz essa afirmação. Outras hipóteses para a diferença de gravidade da doença litiásica seriam decorrentes das características antropométricas, da distribuição de gordura corporal e do limiar de dor ${ }^{2}$.

A abordagem por via laparoscópica constituiu a maior parte dos casos, tanto na análise geral $(61,7 \%)$ quanto na estratificação por sexo $(63,9 \%$ no feminino e 51,3\% no masculino). Desde a sua introdução no final da década de 80 , a literatura já mostra essa tendência, sendo a vídeo-cirurgia o método para tratamento da doença litiásica em 86,6 a 95,5\% dos casos ${ }^{9-10}$. Quando comparada à escolha da via de acesso entre os sexos, entretanto, houve uma diferença estatisticamente significativa $(\mathrm{p}=0,0014)$ para a laparoscópica nas mulheres. No estudo de Golden $e t a l^{9}$ a proporção da cirurgia laparoscópica como opção inicial nos homens mostrou-se menor que o índice global, com $82,2 \%$ dos casos. Se considerarmos os percentuais descritos na literatura, podemos observar que o número de operações por vídeo cirurgia nesta amostra é inferior. Isso pode ser explicado por questões estruturais do serviço, que vão desde a disponibilidade de equipamento até a necessidade de treinamento para os médicos residentes em ambas técnicas.

O tempo operatório foi significativamente maior no sexo masculino, quando empregada a colecistectomia aberta. Na cirurgia laparoscópica, essa diferença não se repetiu, mostrando um tempo equivalente em ambos os grupos. Numa meta-análise realizada por Shea $e t a l^{7}$, a duração média da operação foi de $89 \pm 24,5$ minutos. O tempo operatório maior que a média da literatura também poderia ser justificado pela curva de aprendizado dos médicos residentes em treinamento.

Das intervenções inicialmente realizadas por acesso laparoscópico, aconteceu a conversão em 26 
pacientes, resultando numa taxa geral de 2,31\%. Quando a comparamos entre os sexos, observamos maior percentual no feminino, embora esta diferença não tenha se mostrado significativa estatisticamente. Este dado é confirmado por outros autores, que também não encontraram diferenças numéricas entre os $\operatorname{sexos}^{10-12}$.

Outros estudos já demonstraram uma maior taxa de conversão da técnica laparoscópica para cirurgia aberta em homens, em idosos e nos casos de colecistite aguda ${ }^{6,8,9,13}$. Russel et $a l^{2}$ demonstraram que, ultrapassada a curva de aprendizado na cirurgia laparoscópica, decrescem as conversões, mas persiste a predominância no sexo masculino. Considerando que a alteração anatômica da vesícula biliar e da tríade portal, decorrente do processo cicatricial e fibrótico local, que ocorrem como conseqüência de uma inflamação grave, é a principal causa de alteração do procedimento, conclui-se que a identificação de fatores prognósticos quanto à gravidade da doença litiásica pode resultar em algumas modificações no seu tratamento ${ }^{8,14}$.

As proporções descritas na literatura variam de 2,9 a $9,6 \%$, com uma média de $5 \% .^{7,8,13,14}$. Percentuais maiores são encontrados quando analisados grupos especiais, como idosos, ${ }^{13-15}$ homens ${ }^{11,13}$ e nos casos de colecistite aguda ${ }^{13,14}$. Num estudo com 318 pacientes com idade acima de 65 anos submetidos a colecistectomia laparoscópica, Golden et al ${ }^{9}$ descreveram $20,1 \%$ de conversões que se eleva para 26,5\% quando selecionados apenas os pacientes do sexo masculino. A maior incidência foi observada em homens com colecistite aguda $(40,4 \%)$, com diferença estatisticamente significante quando comparada com a doença aguda nas mulheres $(19,2 \%)$. Essa diferença não se repetiu nos casos de doença crônica, nem quando estratificados os grupos por idade. Além do sexo, idade e história de colecistite aguda, Sanabria et $a l^{8}$ identificaram também como causa para mudança da via operatória: episódios pregressos de cólica biliar e experiência do cirurgião com procedimentos laparoscópicos, e demonstraram que a análise dos fatores de risco poderia prever a conversão em $63 \%$ dos casos no pré-operatório. Assim sendo, pacientes homens com idade superior a 65 anos, com história de colecistite aguda e mais de 10 episódios de cólica biliar têm uma probabilidade de conversão superior a $30 \%$, se submetidos a procedimento laparoscópico. Baseado nestes dados, Sanabria et al ${ }^{8}$ sugerem que para médicos residentes ou cirurgiões em estágios iniciais de treinamento sejam selecionados pacientes de baixo risco, reservando os de alto risco para os mais habilitados, ou seja dotados de maior experiência em colecistectomias laparoscópicas.

Essa tendência da literatura de maiores taxas de conversão no sexo masculino, que não se confirmou em nosso estudo, poderia talvez se justificar pela seleção empírica dos doentes potencialmente complicados para via aberta.

Em relação às causas para a mudança do acesso, a dificuldade técnica foi a mais freqüente $(50 \%)$. Esse dado é corroborado por Shea $e^{2} a l^{6}$, onde $55 \%$ dos casos atribui-se a problemas técnicos, entre os quais citam-se aderências e inflamação. Um dado importante é que apenas $11,5 \%$ das modificações ocorreram devido à lesão de estruturas adjacentes, que neste estudo foram constituídas na sua totalidade por lesões de colédoco, compatível com a literatura que aponta índices inferiores a $15 \%,{ }^{6}$ embora outros estudos demonstrem percentuais ainda menores, de $0,3 \%{ }^{14}$. Na série de Sanabria et $a l^{8}$, somando-se os portadores de aderências, com alteração anatômica e os que tiveram sangramento, estes totalizaram $78 \%$ das causas.

O índice de intercorrências neste estudo foi de $3,5 \%$, enquanto outros autores relatam taxas que variam de 1,6 a $14 \%,{ }^{8,10,12,14,16}$ atingindo até $56 \%$ quando analisadas populações específicas, como ido$\operatorname{sos}^{15}$. Entretanto, critérios de inclusão de pacientes, definição de complicação e tipos de estudos muito distintos dificultam uma comparação.

A permanência hospitalar pós-operatória foi de dois dias, contra 1,6 descritos por Shea et $l^{6} \mathrm{em}$ sua meta-análise. Quando estratificado por sexo, no feminino foi de $1,8( \pm 1,6)$ dias e de $2,7( \pm 4,5)$ no masculino, com diferença estatisticamente significante, justificada pela maior incidência de complicações neste último. Houve significância estatística também quando comparadas entre si as vias de acesso $(\mathrm{p}=0,0000)$. $\mathrm{Na}$ laparoscópica não se verificou a diferença entre os sexos. Esses dados indiretamente reforçam a hipótese da seleção de casos desfavoráveis para a operação tradicional e a observação de que os homens apresentam doença mais complexa, operações tecnicamente mais difíceis e, conseqüentemente, com maior tempo de hospitalização pós-operatória.

A mortalidade da colecistectomia laparoscópica é inferior a $1 \%{ }^{10,14}$. Uma letalidade mais elevada é descrita na literatura em relação à população idosa, sendo até cinco vezes maior na população 
acima de 50 anos do que na jovem. O percentual de óbito descrito no estudo de Golden et $\mathrm{l}^{9}$ que incluiu apenas pacientes com idade superior a 65 anos foi de $1,3 \%$, mas outras constatações chegam a índices de até $10 \%{ }^{18}$. O que se observa também é que, tanto no jovem quanto no idoso, o obituário é maior no sexo masculino. $\mathrm{O}$ risco aumentado de letalidade para os homens, na pesquisa de Russell $e t a l^{2}$, não pode ser explicado pela idade, diagnóstico, co-morbidades, ou procedimento cirúrgico a que foram submetidos. Vários autores concordam em dizer que a colecistite aguda seja mais fulminante em homens, e que a doença litiásica sintomática no sexo masculino deva ser abordada de maneira mais agressiva, intervindo antes da necessidade de procedimento de emergência ${ }^{2,417}$.

As maiores incidências de complicações pósoperatórias encontradas neste estudo e corroboradas pela literatura em relação à doença litiásica em homens não contra-indicam a laparoscopia como via de acesso "standard" da colecistectomia. A redução substancial do período de hospitalização associada a um procedimento laparoscópico bem sucedido, assim como a menor necessidade de analgesia pós-operatória e o retorno precoce às atividades, compensariam os casos em que é realizada conversão para o método aberto. Entretanto, sugerimos que a vídeo cirurgia como via inicial nesses pacientes seja reservada para cirurgiões experientes.

As colecistectomias, convencionais e laparoscópicas, são alternativas seguras para a abordagem da litíase vesicular com índices baixos de morbidade e mortalidade, constituindo o sexo masculino um possível fator de risco nas complicações.

$\mathrm{O}$ sexo masculino mostrou incidência mais elevada de complicações nas colecistectomias quando comparado com o feminino, resultando num tempo operatório e período de permanência hospitalar mais prolongados. A necessidade de drenagem bem como a mortalidade apresentaram frequiências maiores nos homens.

\begin{abstract}
Background: To analyze the influence of gender in the incidence of postoperative complications of open and laparoscopic cholecystectomies. Methods: Retrospective study of 1123 patients who underwent open or laparoscopic elective cholecystectomy in the Department of Surgery of Santa Casa de Misericórdia de São Paulo, between January 1997 and January 2001. Postoperative complications have been evaluated comparatively and results have been related to age, ASA, type and duration of surgical procedure, intra-operative accidents, need of drainage, length of postoperative hospitalization, histopatological findings and mortality. Results: There was a predominance offemale sex in the studied group, representing $82.5 \%$ of cases. The mean age was 48,8 years, ranging from 14 to 97 years old. Six hundred and ninety-three (61,7\%) patients underwent laparoscopic procedures. There was a higher proportion of open procedures in men. $(p=0,0014)$. The overall conversion rate was $2.31 \%$, with no difference between sexes, as well as the associated surgical procedures and the occurrence of intra-operative accidents. Duration of procedure, length of postoperative hospitalization, need of drainage, complications and mortality were significantly higher in males. The analysis of the duration of procedure and length of postoperative hospitalization, showed that the statistical difference occurred at the costs of open cholecystectomy group. Conclusions: Male gender was a risk factor for postoperative complications after cholecystectomies, resulting in a longer duration of procedure and length of postoperative hospitalization, more frequent need of drainage and higher mortality when compared to female gender.
\end{abstract}

Key Words: Cholecystectomy; Postoperative complications; Sex.

\section{REFERÊNCIAS}

1. McSherry CK - Advantages of elective surgical treatment of gallstones. Hepatogastroenterology, 1989, 36(5):330-332.

2. Russell JC, Walsh SJ, Reed-Fourquet L, et al. Symptomatic cholelithiasis: a different disease in men? Connecticut Laparoscopic Cholecystectomy Registry. Ann Surg, 1998, 227(2):195-200.
3. Kumar A, Saxena R, Kapoor VK, et al. Cholecystectomy in high risk patients. Trop Gastroenterol, 1996, 17(1):53-56.

4. Glenn F, Dillon LD - Developing trends in acute cholecystitis and choledocholithiasis. Surg Gynecol Obstet, 1980, 151(4):528-532.

5. MacDonald JA - Early cholecystectomy for acute cholecystitis. Can Med Assoc J, 1974, 111(8):796-799. 
6. Shea JA, Healey MJ, Berlin JA, et al. - Mortality and complications associated with laparoscopic cholecystectomy. A meta-analysis. Ann Surg, 1996, 224(5): 609-620.

7. Shea JA, Berlin JA, Bachwich DR, et al. - Indications for and outcomes of cholecystectomy: a comparison of the pre and postlaparoscopic eras. Ann Surg, 1998, 227(3):343-350.

8. Sanabria JR, Gallinger S, Croxford R, et al. - Risk factors in elective laparoscopic cholecystectomy for conversion to open cholecystectomy. J Am Coll Surg, 1994, 179(6):696-704.

9. Golden WE, Cleves MA, Johnston JC - Laparoscopic cholecystectomy in the geriatric population. J Am Geriatr Soc, 1996, 44(11):1380-1383.

10. Soper NJ, Stockmann PT, Dunnegan DL, et al. Laparoscopic cholecystectomy. The new "gold standard"? Arch Surg, 1992, 127(8):917-921.

11. Liu CL, Fan ST, Lai EC, et al. - Factors affecting conversion of laparoscopic cholecystectomy to open surgery. Arch Surg, 1996, 131(1):98-101.

12. Buanes T, Mjaland $\mathrm{O}$ - Complications in laparoscopic and open cholecystectomy: A prospective comparative trial. Surg Laparosc Endosc, 1996, 6(4):266-272.

13. Fried GM, Barkun JS, Sigman HH, et al. - Factors determining conversion to laparotomy in patients undergoing laparoscopic cholecystectomy. Am J Surg, 1994, 67(1):35-39.
14. Orlando R, Russell JC, Lynch J, et al. - Laparoscopic cholecystectomy. A statewide experience. The Connecticut Laparoscopic Cholecystectomy Registry. Arch Surg, 1993, 128(5):494-498.

15. Uecker J, Adams M, Skipper K, et al. - Cholecystitis in the octogenarian: is laparoscopic cholecystectomy the best approach? Am Surg, 2001, 67(7):637-640.

16. Clavien PA, Sanabria JR, Mentha G, et al. - Recent results of elective open cholecystectomy in a North American and a European Center. Comparison of complications and risk factors. Ann Surg, 1992, 216(6):618-626.

17. Margiotta SJ, Horwitz JR, Wilis IH, et al. Cholecystectomy in the elderly. Am J Surg, 1988, 156(6):509-512.

Endereço para Correspondência:

Dr. Adhemar M. Pacheco Jr.

Área I - Grupo de Vias Biliares e Pâncreas do Departamento de Cirurgia da Faculdade de Ciências Médicas da Santa Casa de São Paulo

Rua Cesário Mota Júnior, 112

CEP 01221-020 - São Paulo - SP

Fone: (11)3226-7271/Fax: (11)3337-8164

E-mail: cir.pancreas@ santacasasp.org.br 